\title{
Basic science: (May 2009)
}

1. Ahmed S, Thomas G, Ghoussaini M, Healey CS, Humphreys MK, Platte R, Morrison J, Maranian M, Pooley KA, Luben R, Eccles D, Evans DG, Fletcher O, Johnson N, Silva ID, Peto J, Stratton MR, Rahman N, Jacobs K, Prentice R, Anderson GL, Rajkovic A, Curb JD, Ziegler RG, Berg CD, Buys SS, McCarty CA, Feigelson HS, Calle EE, Thun MJ, Diver WR, Bojesen S, Nordestgaard BG, Flyger H, Dork T, Schurmann P, Hillemanns P, Karstens JH, Bogdanova NV, Antonenkova NN, Zalutsky IV, Bermisheva M, Fedorova S, Khusnutdinova E, Kang D, Yoo KY, Noh DY, Ahn $\mathrm{SH}$, Devilee P, van Asperen CJ, Tollenaar R, Seynaeve C, Garcia-Closas M, Lissowska J, Brinton L, Peplonska B, Nevanlinna H, Heikkinen T, Aittomaki K, Blomqvist C, Hopper JL, Southey MC, Smith L, Spurdle AB, Schmidt MK, Broeks $A$, van Hien RR, Cornelissen S, Milne RL, Ribas G, Gonzalez-Neira A, Benitez J, Schmutzler RK, Burwinkel B, Bartram CR, Meindl A, Brauch $H$, Justenhoven $\mathrm{C}$, Hamann $U$, Chang-Claude J, Hein R, Wang-Gohrke S, Lindblom A, Margolin $S$, Mannermaa A, Kosma VM, Kataja V, Olson JE, Wang XS, Fredericksen Z, Giles GG, Severi G, Baglietto L, English DR, Hankinson SE, Cox DG, Kraft P, Vatten LJ, Hveem K, Kumle M, et al. Newly discovered breast cancer susceptibility loci on 3p24 and 17q23.2. Nat Genet 2009; 41: 585-590.

2. Caldas-Lopes E, Cerchietti L, Ahn JH, Clement CC, Robles Al, Rodina A, Moulick K, Taldone T, Gozman A, Guo Y, Wu N, de Stanchina E, White J, Gross SS, Ma Y, Varticovski L, Melnick A, Chiosis G. Hsp90 inhibitor PU-H71, a multimodal inhibitor of malignancy, induces complete responses in triple-negative breast cancer models. Proc Natl Acad Sci USA 2009; 106: 8365-8373.

3. Cardamone MD, Bardella C, Gutierrez A, Di Croce L, Rosenfeld MG, Di Renzo MF, De Bortoli M. ER alpha as ligand-independent activator of $\mathrm{CDH}-1$ regulates determination and maintenance of epithelial morphology in breast cancer cells. Proc Natl Acad Sci U S A 2009; 106: 7420-7425.

4. Crowder RJ, Phommaly C, Tao Y, Hoog J, Luo JQ, Perou CM, Parker JS, Miller MA, Huntsman DG, Lin L, Snider J, Davies SR, Olson JA, Watson MA, Saporita A, Weber JD, Ellis MJ. PIK3CA and PIK3CB inhibition produce synthetic lethality when combined with estrogen deprivation in estrogen receptor-positive breast cancer. Cancer Res 2009; 69: 3955-3962.

5. Elsheikh SE, Green AR, Rakha EA, Powe DG, Ahmed RA, Collins HM, Soria D, Garibaldi JM, Paish CE, Ammar AA, Grainge MJ, Ball GR, Abdelghany MK, Martinez-Pomares L, Heery DM, Ellis IO. Global histone modifications in breast cancer correlate with tumor phenotypes, prognostic factors, and patient outcome. Cancer Res 2009; 69: 3802-3809.

6. Fan P, Yue W, Wang JP, Aiyar S, Li Y, Kim TH, Santen RJ. Mechanisms of resistance to structurally diverse antiestrogens differ under premenopausal and postmenopausal conditions: evidence from in vitro breast cancer cell models. Endocrinology 2009; 150: 2036-2045.

7. Feng LY, Ou ZL, Wu FY, Shen ZZ, Shao ZM. Involvement of a novel chemokine decoy receptor CCX-CKR in breast cancer growth, metastasis and patient survival. Clin Cancer Res 2009; 15: 2962-2970.

8. Giamas G, Castellano L, Feng Q, Knippschild $\mathrm{U}$, Jacob J, Thomas RS, Coombes RC, Smith CL, Jiao LR, Stebbing J. CK1 modulates the transcriptional activity of ER via AIB1 in an estrogen-dependent manner and regulates ERAIB1 interactions. Nucleic Acids Res 2009; 37: 3110-3123.

9. Haines E, Minoo P, Feng Z, Resalatpanah N, Nie XM, Campiglio M, Alvarez L, Cocolakis E, Ridha M, Di Fulvio M, Gomez-Cambronero J, Lebrun JJ, Ali S. Tyrosine phosphorylation of Grb2: role in prolactin/epidermal growth factor cross talk in mammary epithelial cell growth and differentiation. Mol Cell Biol 2009; 29: 2505-2520. 
10. Heikkinen T, Karkkainen $\mathrm{H}$, Aaltonen K, Milne RL, Heikkila P, Aittomaki K, Blomqvist C, Nevanlinna $\mathrm{H}$. The breast cancer susceptibility mutation PALB2 1592delT is associated with an aggressive tumor phenotype. Clin Cancer Res 2009; 15: 3214-3222.

11. Hiscox $S$, Jordan $N J$, Smith $C$, James $M$, Morgan L, Taylor KM, Green TP, Nicholson RI. Dual targeting of Src and ER prevents acquired antihormone resistance in breast cancer cells. Breast Cancer Res Treat 2009; 115: 57-67.

12. Lagadec C, Meignan S, Adriaenssens E, Foveau B, Vanhecke E, Romon R, Toillon RA, Oxombre $\mathrm{B}$, Hondermarck $\mathrm{H}$, Le Bourhis $\mathrm{X}$. TrkA overexpression enhances growth and metastasis of breast cancer cells. Oncogene 2009; 28: 1960-1970.

13. Lanigan F, McKiernan E, Brennan DJ, Hegarty S, Millikan RC, McBryan J, Jirstrom K, Landberg G, Martin F, Duffy MJ, Gallagher WM. Increased claudin-4 expression is associated with poor prognosis and high tumour grade in breast cancer. Int J Cancer 2009; 124: 2088-2097.

14. Lott ST, Chen NY, Chandler DS, Yang QF, Wang L, Rodriguez M, Xie HY, Balasenthil S, Buchholz TA, Sahin AA, Chaung K, Zhang BL, Olufemi SE, Chen JY, Adams H, Band V, ElNaggar AK, Frazier ML, Keyomarsi K, Hunt KK, Sen S, Haffty B, Hewitt SM, Krahe R, Killary AM. DEAR1 is a dominant regulator of acinar morphogenesis and an independent predictor of local recurrence-free survival in early-onset breast cancer - art. no. e1000068. PLoS Med 2009; 6: e1000068.

15. Moraes RC, Chang $\mathrm{H}$, Harrington $\mathrm{N}$, Landua JD, Prigge JT, Lane TF, Wainwright BJ, Hamel PA, Lewis MT. Ptch1 is required locally for mammary gland morphogenesis and systemically for ductal elongation. Development 2009; 136: 1423-1432.

16. Qin L, Liu ZL, Chen HW, Xu JM. The steroid receptor coactivator-1 regulates twist expression and promotes breast cancer metastasis. Cancer Res 2009; 69: 3819-3827.

17. Rose-Hellekant TA, Skildum AJ, Zhdankin O, Greene AL, Regal RR, Kundel KD, Kundel DW. Short-term prophylactic tamoxifen reduces the incidence of antiestrogen-resistant/estrogen receptor-positive/progesterone receptor-negative mammary tumors. Cancer Prev Res 2009; 2: $496-502$.

18. Saha T, Rih JK, Rosen EM. BRCA1 downregulates cellular levels of reactive oxygen species. FEBS Lett 2009; 583: 1535-1543.

19. Setiawan VW, Monroe KR, Wilkens LR, Kolonel LN, Pike MC, Henderson BE. Breast cancer risk factors defined by estrogen and progesterone receptor status. Am J Epidemiol 2009; 169: 1251-1259.

20. Sikora MJ, Cordero KE, Larios JM, Johnson MD, Lippman ME, Rae JM. The androgen metabolite 5 alpha-androstane-3 beta, 17 beta-diol ( 3 beta Adiol) induces breast cancer growth via estrogen receptor: implications for aromatase inhibitor resistance. Breast Cancer Res Treat 2009; 115: 289-296.

21. Soni S, Lin BT, August A, Nicholson RI, Kirsch $\mathrm{KH}$. Expression of a phosphorylated p130(Cas) substrate domain attenuates the phosphatidylinositol 3-kinase/akt survival pathway in tamoxifen resistant breast cancer cells. J Cell Biochem 2009; 107: 364-375.

22. Thomas $G$, Jacobs $K B$, Kraft $P$, Yeager $M$, Wacholder S, Cox DG, Hankinson SE, Hutchinson A, Wang Z, Yu K, Chatterjee N, Garcia-Closas M, Gonzalez-Bosquet J, Prokunina-Olsson L, Orr N, Willett WC, Colditz GA, Ziegler RG, Berg CD, Buys SS, McCarty CA, Feigelson HS, Calle EE, Thun MJ, Diver R, Prentice R, Jackson R, Kooperberg C, Chlebowski R, Lissowska J, Peplonska B, Brinton LA, Sigurdson A, Doody $\mathrm{M}$, Bhatti $\mathrm{P}$, Alexander $\mathrm{BH}$, Buring J, Lee IM, Vatten LJ, Hveem K, Kumle M, Hayes RB, Tucker $M$, Gerhard DS, Fraumeni JF, Hoover RN, Chanock SJ, Hunter DJ. A multistage genomewide association study in breast cancer identifies two new risk alleles at 1p11.2 and 14q24.1 (RAD51L1). Nat Genet 2009; 41: 579-584.

23. Udler MS, Meyer KB, Pooley KA, Karlins E, Struewing JP, Zhang J, Doody DR, MacArthur S, Tyrer J, Pharoah PD, Luben R, Bernstein L, Kolonel LN, Henderson BE, Le Marchand L, Ursin G, Press MF, Brennan P, Sangrajrang S, Gaborieau V, Odefrey F, Shen CY, Wu PE, Wang HC, Kang D, Yoo KY, Noh DY, Ahn SH, Ponder BAJ, Haiman CA, Malone KE, Dunning AM, Ostrander EA, Easton DF. FGFR2 variants and breast cancer risk: fine-scale mapping using African American studies and analysis of chromatin conformation. Hum Mol Genet 2009; 18: 1692-1703.

24. Zhang Y, Martens JWM, Yu JX, Jiang J, Sieuwerts AM, Smid M, Klijn JGM, Wang YX, Foekens JA. Copy number alterations that predict metastatic capability of human breast cancer. Cancer Res 2009; 69: 3795-3801.

Prepared by

$R$ Sutherland

Cancer Research Program

Garvan Institute of Medical Research Darlinghurst, NSW, Australia 